\title{
SISTEMA DE INDICADORES DE GESTIÓN EN LA CALIDAD Y PRODUCTIVIDAD EN LA DOCENCIA UNIVERSITARIA
}

\section{SYSTEM MANAGEMENT INDICATORS IN QUALITY AND PRODUCTIVITY IN UNIVERSITY TEACHING}

\author{
José V. Sánchez-F. ${ }^{1}$, Héctor Fabian Parra-López²
}

Forma de citar: SÁNCHEZ-F. José, PARRA-LÓPEZ Héctor. Sistema de indicadores de gestión en la calidad y productividad en la docencia universitaria. Eco.Mat. 2014; 5(1): 102 - 116.

Recibido:

Agosto 20 de 2014

Aceptado:

Octubre 22 de 2014

${ }^{1}$ Licenciado en Educación Mención Física y Matemática, Magister Scientarum Estadística, Doctor en Educación. Exrector Universidad Experimental del

Táchira UNET, San Cristóbal -

Venezuela. jusfrank@gmail.com

${ }^{2}$ Abogado, Universidad Externado de Colombia especialista en derecho público, Universidad Externado de Colombia, Especialista en derecho electoral, universidad de rosario, Msc Derecho público universidad Carlos III España; Candidato a Doctor Universidad de granada España. Correo: hfabianparrac@hotmail.com.

\section{RESUMEN}

La investigación objeto de este trabajo desarrolla los aspectos teóricos de los constructos calidad y la productividad en la docencia universitaria. Plantea como objetivo general diseñar e implementar un sistema de medición de la calidad y productividad en la docencia universitaria, con base en las nuevas tecnologías de la información y la comunicación (NTIC), utilizando indicadores, con aplicación en ambiente Web. La investigación combina los dos enfoques tradicionales, el paradigma cuantitativo con la finalidad de conseguir patrones de regularidad, responder a las preguntas de investigación y comprobar las hipótesis, y el cualitativo en cuanto a nivel de técnica y estrategia de captura de información, con las entrevistas a profundidad, y también a nivel del análisis de datos, e interpretación de los resultados en la reducción, disposición y transformación de los mismos, a los fines de obtener de la experiencia de los cuatro grupos etarios de educadores; conforme a lo contemplado en la tesis de la diversidad complementaria de los diferentes paradigmas de investigación de las ciencias sociales y la educación. Los resultados en las dos metodologías se utilizan conjuntamente para el diseño e implementación de un sistema de medición de la calidad y la productividad en la docencia, apoyado en las nuevas tecnologías de la información y la comunicación, en ambiente Web, utilizando indicadores.

La implementación del sistema permitirá ayudar en la formulación, ejecución y seguimiento de programas, orientados a la formación del personal académico para contribuir a mejorar la calidad y productividad de la docencia en la universidad.

Palabras claves: docencia, calidad, productividad, sistema e indicadores.

\section{SUMMARY}

The research for this paper develops the theoretical aspects of the constructs quality and productivity in university teaching. The general 
objective to design and implement a system for measuring the quality and productivity in university teaching, based on the new technologies of information and communication (NTIC), using indicators, Web application environment. The research combines the two traditional approaches, the quantitative paradigm in order to get regular patterns, answer the research questions and test hypotheses, and in terms of the qualitative level of technique and strategy of capturing information, interviews with depth, and level of data analysis, and interpretation of the results in the reduction, disposal and processing of the same, in order to get experience of the four age groups of educators; provided in accordance with the thesis of the complementary diversity of different research paradigms of the social sciences and education. The results in the two methodologies are used together to design and implement a system for measuring quality and productivity in teaching, supported by the new information technologies and communication in Web environment using indicators.

The implementation of the system will help in the formulation, implementation and monitoring of programs aimed at training of academic staff to help improve the quality and productivity of teaching at the university.

Keywords: teaching, quality, productivity, and system indicators

\section{INTRODUCCIÓN}

$\mathrm{E}$ 1 tema de la calidad y productividad en la educación es de sumo interés en toda la sociedad. La universidad en el contexto universal, nacional y local, ha evidenciado la preocupación por estos temas, comenzando a dar muestras de la necesidad de una medición y evaluación institucional cualicuantitativa de sus entradas, insumos, procesos, productos y resultados de las funciones docencia, investigación, extensión y gestión institucional, reconociendo la necesidad de generar sistemas de medición con base en las nuevas tecnologías de la información y la comunicación (NTIC), utilizando indicadores, con aplicación en ambiente Web.

En este trabajo se realizó el diagnóstico y evaluación de los dispositivos desarrollados para la medición de la calidad y productividad en la docencia, con el propósito de diseñar e implementar un sistema de medición con base en las NTIC, utilizando indicadores, con aplicación en ambiente Web, se concibió la investigación como un sistema, en consecuencia, con un enfoque sistémico para sustentarla; por un lado el paradigma cuantitativo analizó la realidad externa, el uso del paradigma cualitativo, para la realidad interna, además de la sinergia propia del enfoque sistémico, que generó la solidez de este trabajo.

La investigación es fundamental en la docencia, se inscribe en la conceptualización moderna del docente universitario al concebirlo no como un simple trasmisor de conocimientos (lo que es), sino como un sujeto que tiene de soporte a la función investigación, lo que se conoce con el nombre de docente-investigador (lo que debe ser).

Este primer obstáculo, permite visualizar un panorama de poca claridad, para iniciar procesos de transformación en la búsqueda de mejorar la calidad y productividad en
ISSN 1794-8231

PP: $102-116$ 
No. 1

Enero-Diciembre 2014 ISSN 1794-8231

PP: $102-116$ la docencia; y la carencia casi total de un dispositivo para medir y monitorear estos dos constructos y su mejoramiento (lo que es), lo cual hace imperioso diseñar un sistema, con base en las NTIC, utilizando un conjunto de indicadores de gestión que permita medir cómo se avanza por ese camino (lo que debe ser), apuntalando el mejoramiento continuo en esta función básica formulando una serie de preguntas que sirvieron de guía a la investigación, aquí mostramos algunas: 1.¿Cuáles son los elementos que deben conformar la función docencia? 2. ¿Se dispone de algún mecanismo que permita medir en forma sistemática, la calidad y productividad en la docencia? 3. ¿Cuál es la proporción de profesores que consideran a las NTIC como una herramienta para mejorar la calidad en la docencia? 4. ¿Cuál es la proporción de profesores que consideran a las NTIC como una herramienta para mejorar la productividad en la docencia? 5. ¿Qué proporción de profesores conoce indicadores de calidad en la docencia? 6. ¿Qué proporción de profesores conoce indicadores de productividad en la docencia? 7. ¿Qué tanto mejora la calidad en la docencia de los profesores contratados que han sido sometidos a la intervención con respecto a los profesores ordinarios? 8. ¿Qué tanto mejora la productividad en la docencia de los profesores contratados que han sido sometidos a la intervención con respecto a los profesores ordinarios? 9. ¿Cuál es la proporción de estudiantes que considera se debe contar con un sistema para medir la calidad en la docencia? 10. ¿Cuál es la proporción de estudiantes que considera se debe contar con un sistema para medir la productividad en la docencia? 11. ¿Cuáles indicadores pueden plantearse para medir la calidad en la docencia? 12. ¿Cuáles indicadores pueden plantearse para medir la productividad en la docencia? 13. ¿Cuál plan de mejora puede surgir de medir la calidad y la productividad en la docencia?

Estas preguntas generaron los objetivos y las hipótesis que guiaron la investigación:

\section{Objetivo General}

Implementar un sistema con base en las nuevas tecnologías de la información y la comunicación (NTIC), para la medición de la calidad y productividad en la docencia universitaria, utilizando indicadores.

\section{Hipótesis de la Investigación}

Las hipótesis que se enuncian a continuación son de trabajo, luego en la operacionalización se trasforman en hipótesis estadísticas (expresiones matemáticas) afirmaciones concretas sobre los parámetros de la población. Se menciona algunas de ellas:

1. Una pequeña proporción de profesores conoce los elementos que deben conformar la función docencia.

2. Hay diferencias significativas entre la proporción de profesores contratados y la proporción de profesores ordinarios en cuanto al conocimiento de los componentes de la función docencia

3. Una gran proporción de profesores afirma que la institución carece de dispositivos que permitan medir en forma sistemática la calidad y productividad en la docencia.

4. Una gran proporción de profesores considera a las NTIC como una herramienta para mejorar la calidad en la docencia.

5. Una gran proporción de profesores considera a las NTIC como una herramienta para mejorar la productividad en la docencia.

6. Existen diferencias significativas entre la proporción de profesores contratados $\mathrm{y}$ la proporción de profesores ordinarios que consideran a las NTIC como una herramienta para mejorar la calidad en la docencia.

7. Existen diferencias significativas entre la proporción de profesores contratados y la proporción de profesores ordinarios que consideran a las NTIC son una herramienta para mejorar la productividad en la docencia. 
8. Una pequeña proporción de profesores de la universidad conoce indicadores de calidad en la docencia.

9. Una pequeña proporción de profesores de la universidad conoce indicadores de productividad en la docencia.

10. La calidad en la docencia de los profesores contratados que han sido sometidos a la intervención (cursos de la coordinación de desarrollo educativo) es mejor a la de profesores ordinarios.

11. La productividad en la docencia de los profesores contratados que han sido sometidos a la intervención es mejor a la de profesores ordinarios.

Las hipótesis número 1 a 11 fueron probadas con los análisis de los instrumentos de diagnóstico que se aplicaron a los profesores y estudiantes; y las número 12 y 13 con la implementación del sistema de medición en la muestra en el departamento seleccionado.

\section{MARCO EPISTEMOLÓGICO}

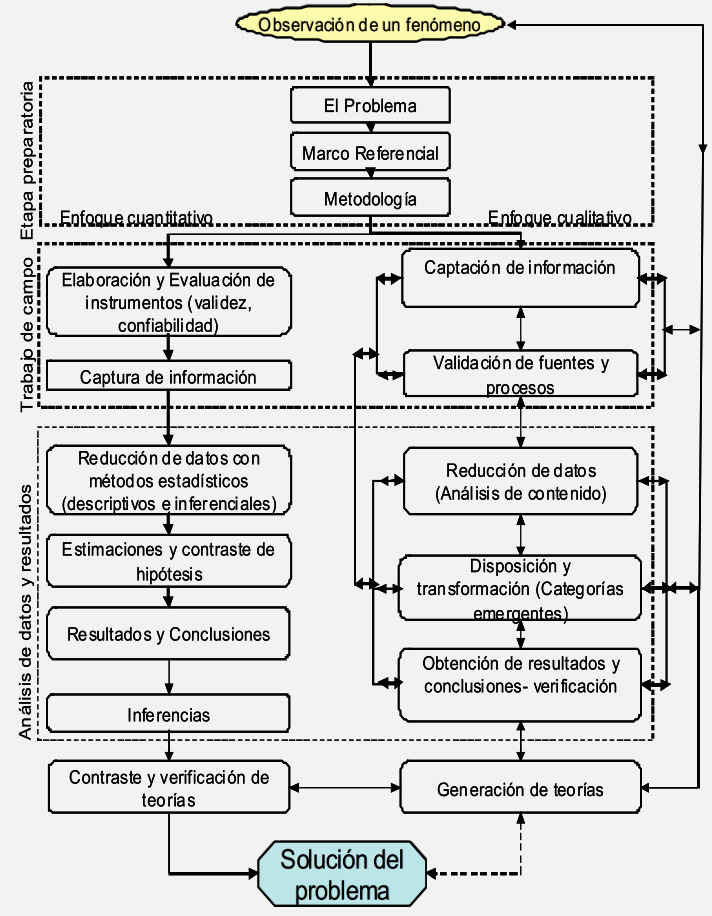

Gráfico1. Esquema de aplicación del Teorema de la Diversidad Complementaria. Construcción original del autor.

\section{Teorema de la Diversidad Complementaria}

La investigación se aborda desde los paradigmas: cuantitativo (epistemológica y metodológicamente), y cualitativo (nivel técnico, análisis de la información, y generación e interpretación de resultados); con base en la tesis de la diversidad complementaria de los diferentes paradigmas de investigación de las ciencias sociales y la educación, defendida y legitimada por diversos investigadores Cook y Campbell (1979), y otros.

La superación del debate metodológico cualitativo/cuantitativo definido por Martínez (1999) y Rusque (1999), la unidad metodológica en la investigación científica planteada por Cerda (2001) y, la tesis de la complementariedad por deficiencia planteada por Padrón (2000).

Adicionalmente en esta investigación como una argumentación más que justifica la complementariedad de los dos enfoques, dado que el objeto de estudio, la calidad y la productividad en la docencia universitaria, se puede descontextualizar de la estructura o sistema social, sin que pierda su esencia o desvirtúe su naturaleza (Martínez, 1999), que hacen obligatorio el uso del enfoque cuantitativo y de técnicas de análisis matemáticas y estadísticas basadas en la probabilidad.

Por otro lado dicho objeto de estudio o fenómeno es posible abordarlo con el enfoque cualitativo, a los niveles ya mencionados; en el gráfico 1 ilustra el planteamiento.

\section{Marco Metodológico}

\section{Tipo de Investigación}

De acuerdo a su finalidad, es aplicada, ya que se dirige a contribuir en la resolución de un problema relacionado con la ausencia de buenos dispositivos para la medición de la calidad y productividad en la docencia universitaria. Según el nivel, que viene dado por el grado de profundidad y alcance, se adoptará la clasificación propuesta por
Enero-Diciembre 2014 ISSN 1794-8231 PP: $102-116$ 
No. 1

Padrón (2000), de la cual participa el autor, que considera cuatro tipos: descriptiva, explicativa, contrastiva y aplicativa.

Esta investigación llega al nivel de contrastiva, permiten un estudio de campo, método experimental, replicaciones de comprobación de hipótesis variando las condiciones de la prueba, con grupo control y experimental, para hacer estudios y análisis similares, pero con mayor profundidad que las explicativas.

\section{Diseño de la Investigación}

Este trabajo corresponde a un diseño de campo donde los datos de interés para el diseño del sistema de medición se recogieron directamente de la realidad, mediante el trabajo concreto (muestreo aleatorio estratificado en profe-sores, alumnos; y muestreo intencional de expertos en el área), El diseño que se utiliza en esta investigación es similar al diseño 6 de Campbell y Stanley (1995), con prueba posterior y grupo control, que en el caso de los cuasiexperimentales se denomina diseño sólo con pos prueba y grupos intactos (Hernández, Fernández \& Baptista, 1999); y comparación con grupo estático o intacto (Salkind, 1999), que significa no asignados aleatoriamente a los grupos, por estar ellos ya definidos.

\section{Definición de Variables}

Con relación al paradigma cuantitativo las variables que fueron consideradas en esta investigación son: las que controló el investigador, las independientes, entre las que se incluyeron el dispositivo de medición, los alumnos, los profesores, las asignaturas, dependientes las que se midieron: docencia el uso tecnologías, calidad y productividad. Las definiciones conceptuales y operacionales están en el marco teórico, y en los cuadros de operacionalización de las variables e hipótesis.

\section{Población, Muestra y Muestreo}

En el caso de las poblaciones de los profesores y alumnos la forma de muestrear fue sin reemplazo, del tipo semiprobabilístico

inferior, ya que los profesores estaban previamente asignados por su condición de ordinario o contratado y los alumnos similarmente a sus carreras donde existen profesores de ambas condiciones, y asignados a los departamentos docentes, y la técnica estratificado con afijación proporcional; en el enfoque cuantitativo; y la muestra intencional en expertos en el área y por grupos etarios, en el enfoque cualitativo.

\section{Instrumentos de Captación de Información}

La información para el enfoque cuantitativo fue recabada de las encuestas aplicadas a la muestra aleatoria de profesores y alumnos. Se elaboraron dos encuestas diagnóstico (instrumentos), una que se aplicó a los profesores y otra a los estudiantes.

La primera sección, corresponde al perfil psicográfico del entrevistado; la segunda, se trata de determinar la concepción que tienen los profesores de la docencia; la tercera sección, trató de averiguar la postura que sobre la calidad en la docencia tenían los encuestados, cómo la relaciona con las NTIC y con las actividades de docencia. En las restantes secciones, de manera similar, se trató de investigar su posición con relación a la productividad, eficiencia, efectividad y eficacia, cómo maneja el profesor su relación de estos tópicos con las NTIC, y los sistemas de medición de calidad y productividad en la docencia; las dos últimas secciones de la encuesta trataron con los indicadores de calidad y productividad en la docencia.

La encuesta diagnóstico aplicada a los estudiantes estuvo referida a las competencias y perfil que posee el docente (lo que es), y las competencias y perfil que ellos piensan debe poseer (lo que debe ser) el docente en la universidad.

En cuanto al paradigma cualitativo los instrumentos para recolectar la in-formación estuvieron constituidos por entrevistas semiestructuradas a profundidad con un guión, el cual contempló algunos tópicos 
denominados categorías referenciales; se incluyeron aspectos como la concepción de la docencia, la calidad en la docencia, la productividad en la docencia, los sistemas de medición con base en las NTIC utilizando indicadores.

\section{Confiabilidad y Validez de los Instrumentos y los Resultados}

Confiabilidad y validez. La con-fiabilidad en el enfoque cuantitativo utilizada para los instrumentos (encuesta piloto y encuesta diagnostico) y los resultados fue de la consistencia interna como medida de homogeneidad, se hizo por constructo $y$ general, con intervalos de confianza utilizando el coeficiente $\alpha$ de Cronbach.

En el enfoque cuantitativo: la validez utilizada fue la aparente y de contenido realizada a través de expertos en contenido y en elaboración de instrumentos; y la validez de constructo utilizando análisis de factores. En el enfoque cualitativo: la confiabilidad se midió por el grado de coincidencia en el contexto de los evaluadores y el evaluado; la validez mediante la triangulación que en diferentes tipos (datos, métodos, fuentes de información, tiempo, entre otras).

\section{Fundamento Teórico del Análisis de los Datos y Resultados}

Se entenderá para efectos de este trabajo el análisis de datos como lo definen Rodríguez, Gil y García (1999) "un conjunto de manejos, transformaciones, operaciones, reflexiones y comprobaciones que se realizan sobre los datos" (p. 200), con el fin de extraer conclusiones, inferencias y significados relevantes con respecto a un problema objeto de investigación.

\section{Análisis de datos y resultados en el enfoque cuantitativo}

Los análisis estadísticos realizados fueron de tipo descriptivo e inferencial. Primero, descriptivos se incluyeron para la organización y presentación de la información, en diferentes formatos: a) formato gráfico que incluye diagramas de líneas; b) tablas de distribución de frecuencia, porcentajes; y c) analítica, cálculos estadísticos básicos y estimadores, que constituyen variables aleatorias o medidas resumen de las muestras, incluyendo medidas de posición o tendencia central y medidas de dispersión, para variables medidas desde la escala nominal hasta la de proporción.

Segundo, inferenciales se realizaron diferentes análisis, así, para escalas nominales se estimaron, medidas de asociación la prueba ji-cuadrada, el coeficiente de Kappa, el coeficiente Kaiser-Meyer-Olkin (KMO); para las escalas de orden superior (ordinal, intervalo y proporción) se incluyeron en los análisis, estimaciones: puntual y por intervalo de otros parámetros, pruebas de hipótesis $\mathrm{y}$ de comparaciones múltiples (para una y dos poblaciones), de variabilidad conjunta o análisis de la varianza univariada (ANAVAR) para más de dos poblaciones cuando sea una sola variable dependiente, pruebas de comparaciones múltiples a posteriori o post hoc análisis de correlaciones de momento de Pesaron, y otras de las técnicas para espacios muestrales multivariados tales como análisis por factores o análisis factorial tradicional, componentes de agrupamiento o para reducción de variables, y el análisis de correspondencia. Para efecto de la parte operacional se utilizó software estadístico.

\section{Análisis de los Datos y Resultados en el Enfoque Cualitativo}

El proceso de análisis de los datos, propiamente tal, prosigue con tres tareas: la reducción de los datos, la disposición y transformación de datos, y obtención y verificación de conclusiones.

La reducción se contempla la separación de unidades; identificación y clasificación; síntesis y agrupamiento de unidades. SN 1794-8231 $102-116$

La disposición y transformación de los datos. En primer lugar la disposición se refiere a la organización de la información ya depurada, clasificada y sintetizada. En segundo lugar la transformación de los datos, 
No. 1

se genera cuando es necesario cambiar el lenguaje utilizado para expresar los datos.

La obtención y verificación de conclusiones. Incluye dos actividades: proceso para obtener resultados y conclusiones, y verificación de las conclusiones.

\section{Análisis De La Información Interpretación De Resultados}

Se presentan los principales hallazgos de la investigación, obtenidos a través del análisis e interpretación de los datos generados de las diferentes fuentes de información: los instrumentos de medición, es decir, las encuestas semi-estructurardas, las encuestas diagnóstico aplicadas a profesores $y$ estudiantes, las encuestas en la Web, las bases de datos de control de estudios y recursos; los análisis para la estimación de la confiabilidad y la validez de los instrumentos; los resultados de los análisis utilizando las técnicas de análisis de contenido, de estadística descriptiva e inferencial, para organizar, presentar la información, y dar respuesta a las preguntas de investigación a través de los contrastes o pruebas de hipótesis.

En primer lugar, se realizó el análisis cualitativo de contenido temáticos provenientes de las entrevistas semiestructuradas, se generaron las categorías y subcategorías, se construyó el mapa temático; y se interpretaron estos resultados; en segundo lugar, los análisis cualicuantitativos de confiabilidad y validez, información proveniente de las encuestas, para la generación de los estimadores cualitativos o juicios, puntuales y por intervalo, con su respectiva interpretación; y en tercer lugar, los análisis cuantitativos de los datos obtenidos en las encuestas, utilizando estadística descriptiva e inferencial, generando: perfiles, estimadores puntuales y por intervalo para los estadísticos de proporción, resultados de contrastes de hipótesis y la interpretación de estos resultados. Ratificando la aplicación teorema de la diversidad complementaria.

\section{Análisis Cualitativo de la Información}

A nivel técnico, de análisis de la información,

y generación e interpretación de resultados, para triangular los resultados de las encuestas diagnóstico y con las entrevistas semiestructuradas, lo cual permitió el diseño (generación de los indicadores) del sistema, de manera más consistente y confiable.

Para efectos del análisis PI es el informante I, con $\mathrm{I}=1,2,3,4$, y 5.

Una vez aplicada la entrevista semiestructurada a los profesores seleccionados, se procedió a realizar una organización de la información, hasta obtener la categorización a partir de los relatos, los cuales generaron los códigos y subcategorías, para dicho análisis e interpretación de los resultados. La secuencia seguida en el tratamiento de los datos cualitativos fue la siguiente:

1. Trascripción textual de la información grabada en los cassettes.

2. Elaboración de la matriz de codificación de unidades temáticas de análisis. Para esto se copiaron los micro actos de habla de los informantes (P1..P5) seccionados en unidades temáticas (párrafos) para asignarles códigos de acuerdo al análisis del texto: Construcción versus transmisión; evaluación de la docencia; docencia versus otras funciones; enseñanza aprendizaje; complejidad de la docencia universitaria; rol facilitador-investigador; pensamiento individualista versus integrador; cantidad y no calidad de la docencia; docencia universitaria; compromiso de la docencia; rol orientador; ópticas para la formulación de indicadores; integración de las funciones universitarias; docencia-investigación; NTIC nuevos paradigmas educativos; rol investigador; elementos básicos para la investigación; formación integradora del universitario; tipos de docentes universitarios; comunicación entre docentes-investigadores; evaluación de las NTIC; integración de las funciones universitarias: causas de la improductividad docente; Investigación educativa; relación docencia investigación; diferenciación contextual de los sistemas de información; la tecnología y el hombre; integración de 
funciones; competencias docentes; docencia tradicional; NTIC y culturas; NTIC-docencia; NTIC y medición tecnológica; confusión entre docencia y enseñanza; NTIC-docencia tradicional; evaluar para mejorar; ausencia de indicadores de calidad; necesidad de indicadores; variabilidad de los indicadores; concepción de la calidad en la docencia; la pertinencia del sistema de evaluación; estímulos de la productividad; entre otros, que corresponden a lo que Rodríguez, Gil y García (1999) denominan reducción de datos ilustrado en el Gráfico 2.

3. La agrupación de los códigos emergieron las 19 subcategorías mostradas en el mapa temático: docencia tradicional; roles de los docentes; integración de funciones; administración de la docencia; acto pedagógico; las cuales generan la categoría Docencia Universitaria. Las subcategorías: tecnologías; tecnologías educativas; docencia universitaria y NTIC, generan la categoría Las NTIC en la Educación. Las subcategorías: calidad institucional; y productividad institucional generan la categoría Calidad y Productividad Universitaria. Por último las subcategorías: necesidad de evaluación; indicadores de evaluación; componentes del sistema; diferenciación tempo-ro-espacial; finalidad de la evaluación; participación de los actores; pertinencia del sistema; resistencia a la evaluación; e indicadores de gestión, generan la categoría Evaluación Universitaria. Se construyó para cada una la matriz de categorías de unidades temáticas de análisis.

4. Elaboración de la matriz de categorías de unidades temáticas de análisis por subcategorías.

5. Elaboración de la matriz de interpretación de categorías. Cada categoría con sus respectivas subcategorías y sus micro actos de habla fueron analizadas horizontalmente para encontrarle sentido y determinar sus tendencias a los fines de su interpretación. Estas matrices son el insumo básico para realizar el informe de resultados, siendo los hallazgos encontrados de gran importancia para reforzar el análisis cuantitativo e interpretación de los resultados de la presente tesis.

Los pasos 3, 4 y 5 corresponden a lo que Rodríguez, Gil y García (1999) denominan disposición y transformación de los datos, ilustrado en el Gráfico 2.

6. Para el análisis de las categorías y subcategorías, la triangulación como estrategia de investigación para validar la propuesta teórica respecto a la concepción de la docencia, calidad y la productividad en la docencia, las NTIC y la educación, la medición y evaluación universitaria en la docencia, así como su interacción que constituye el sistema y la perspectiva sistémica, objetivo y enfoque planteado en esta investigación se afianzó en diferentes tipos de triangulación (Rodríguez, 2005). Las de datos, basada en criterios espaciotemporales; en tiempo, datos recogidos en diferentes momentos y la cual consiste en el escaso conocimiento y utilización de la concepción de estos constructos; en espacio, porque los datos se captaron en poblaciones heterogéneas, aumentado la variedad de los datos y evitando problemas como el sesgo de las unidades de análisis y; triangulación en investigadores, y la triangulación de métodos (Rodríguez, 2005).

7. Elaboración de un mapa temático de categorías o Red de Categorías, el cual muestra en forma gráfica las interacciones de las categorías y las subcategorías con el objeto de estudio de la investigación (ver Gráfico 2).

8. Obtención de conclusiones mediante el análisis centrado en las categorías y subcategorías emergentes.

Los pasos 6,7 y 8 conforman la obtención y verificación de conclusiones.

\section{Análisis Cuantitativo de la Información}

En el Cuadro 1 se presentan los resultados de la estimación puntual y por intervalo de los estadísticos de confiabilidad, para cada uno de los constructos y para la encuesta en general; la estimación de coeficientes por cons-tructo
Enero-Diciembre 2014 ISSN 1794-8231 PP: $102-116$ 
No. 1

Enero-Diciembre 2014 ISSN 1794-8231 PP: $102-116$ es la más conveniente y garantiza mayor fiabilidad de los resultados (Sánchez, 2001). Se utilizaron dos tipos de validez aparente y de contenido, y dos procesos de validación que realizaron a través de expertos, confirmaron un instrumento altamente válido.

La validez de constructo y el proceso de validación se realizó mediante el análisis factorial tradicional, para la muestra completa de profesores (194); utilizando el valor del estadístico Kaiser-Meyer-Olkin (KMO), con una medida de adecuación de la muestra de $.70,80$ y una prueba de esfericidad de Bartlett's con un nivel confianza superior al .99. Los factores resultantes de la matriz rotada ortogonalmente, se seleccionaron ocho factores en función de las dimensiones (constructos) de la encuesta diagnóstico.

Cuadro 1. Coeficientes de Confiabilidad e Intervalos de Confianza Estimados para el Alfa de Cronbach, de la Encuesta Diagnóstico Aplicada a los Profesores

\begin{tabular}{lccccc}
\hline \multirow{2}{*}{ Dimensión } & $\hat{\alpha}$ & $n$ & $k$ & $\alpha_{L}(z)$ & $\alpha_{R}(z)$ \\
\hline Docencia & .9287 & 193 & 22 & .9125 & .9419 \\
Calidad & .7917 & 194 & 10 & .7431 & .8311 \\
Productividad & .8373 & 194 & 11 & .7995 & .8679 \\
Efectividad & .7414 & 194 & 7 & .794 & .7914 \\
Eficiencia & .7607 & 194 & 7 & .7033 & .8070 \\
Eficacia & 7131 & 194 & 5 & .6416 & .7703 \\
\hline Total & .9565 & 193 & 124 & .9469 & .9644 \\
\hline
\end{tabular}

\section{Análisis descriptivo a la encuesta}

\section{Diagnóstico Aplicada a los Profesores}

Encuesta diagnóstico aplicada a los profesores. El análisis descriptivo se inicia a partir de las variables (características) correspondientes, 110 y se presentan en tablas de frecuencia para todas las variables.

Por ejemplo: la opinión de los profesores de acuerdo a su condición laboral, en lo referido a la calidad de la docencia en la universidad; sus sistemas de medición con base en las NTIC y utilizando indicadores, lo cual se consideró en las variables I34 a I38.

En cuanto a la calidad, existen algunas diferencias al considerar su condición laboral de contratados y ordinarios:
1. Variable I34, "la docencia que se imparte es de buena calidad", en las categorías de respuesta, De acuerdo y Totalmente de acuerdo, son similares, para los profesores contratados un $71 \%$ y para los profesores ordinarios un $67 \%$.

2. Variable I35, "existe un dispositivo para la medición de calidad en la docencia", en las categorías de respuesta, En desacuerdo y Totalmente en desacuerdo, existen diferencias sustanciales, para los profesores contratados un $28 \%$ y para los profesores ordinarios un $55 \%$, preocupa que el $40 \%$ de los profesores contratados y el $26 \%$ de los ordinarios prácticamente no opinan o son indiferentes a la situación.

3. Variable I36, "se realiza la medición de calidad en la docencia de manera sistemática", en las categorías de respuesta, En desacuerdo y Totalmente en desacuerdo, existen diferencias sustanciales, para los profesores contratados un $46 \%$ y para los profesores ordinarios un $68 \%$, lo que más preocupa es que el $34 \%$ de los profesores contratados y el $19 \%$ de los ordinarios no opinan prácticamente. Para mencionar algunas de manera similar se hace en todas las variables y en la encuesta diagnóstico aplicada a los alumnos.

\section{Análisis inferencial encuesta diagnóstico aplicada a los profesores.}

Corresponde en esta sección probar las hipótesis, hacer los análisis de varianza (ANAVAR) y de correspondencias múltiples $\mathrm{y}$ totales.

El procedimiento que se utilizó fue la técnica de recategorización con el método de la raíz cuadrada de la frecuencia acumulativa; proceso que consiste, primero, en calcular el promedio de la puntuación asignada a cada categoría de respuesta a partir de las frecuencias de respuesta, en cada una de las categorías para todos los ítems involucrados, este procedimiento se repite para todos los sujetos, obteniendo tantos promedios ponderados como sujetos existentes; segundo, se categorizan utilizando el método 
acumulativo de la raíz cuadrada de la frecuencia (Scheaffer, Mendenhall \& Ott, 1986/1987) obteniendo una variable compactada, que corresponde a la opinión de los encuestados cuando existen varios ítems sobre un constructo o variable original. Estas nuevas categorías se utilizan

Enero-Diciembre 2014 en los análisis.

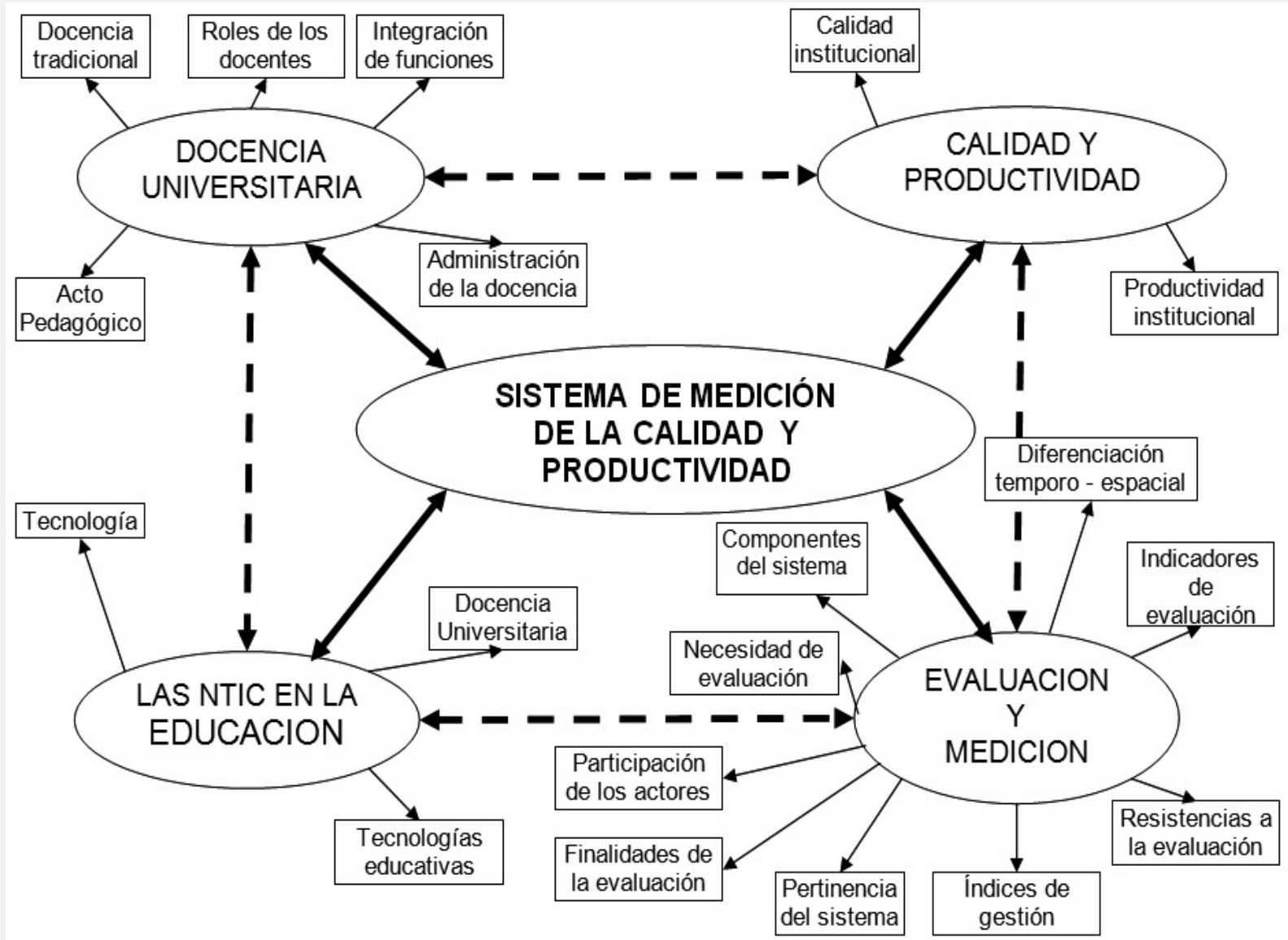

Gráfico 2. Red de categorías. Elaborado con datos e información generada del análisis cualitativo.

Para responder la primera pregunta de investigación, se contrastaron resultados de pruebas de las hipótesis 1 y 2 . Para la hipótesis 1 , se examinó la hipótesis de trabajo inicial "una pequeña proporción de profesores conoce los elementos que deben conformar la función docencia en la universidad de acuerdo lo establecido en la definición, lo que generó la docencia como variable compactada en cinco categorías (ver Cuadro 2).
Cuadro 2. Categorías Variable Docencia Compactada

\begin{tabular}{llll}
\hline $\begin{array}{l}\text { Variable } \\
\text { categórica }\end{array}$ & $\bar{X}$ & Valor & $f$ \\
\hline $\begin{array}{l}\text { Totalmente en } \\
\text { desacuerdo }\end{array}$ & $<4.2$ & 1 & 21 \\
En desacuerdo & $\begin{array}{l}{[4.2 \mathrm{a}} \\
4.4)\end{array}$ & 2 & 36 \\
& $\begin{array}{l}{[4.4 \mathrm{a}} \\
\text { Indeciso }\end{array}$ & 3 & 42 \\
& $\begin{array}{l}4.6) \\
{[4.6 \mathrm{a}}\end{array}$ & 4 & 48 \\
De acuerdo & $\begin{array}{l}4.7) \\
\geq 4.7\end{array}$ & 5 & 47 \\
$\begin{array}{l}\text { Totalmente de } \\
\text { acuerdo }\end{array}$ & & & \\
\hline
\end{tabular}


No. 1

Enero-Diciembre 2014 ISSN 1794-8231

PP: $102-116$
Se sometieron a prueba las hipótesis estadísticas nula y alternativa Ho: $\mathrm{P} \leq .57 \mathrm{y}$ $\mathrm{H}_{1}: \mathrm{P}>.57$, respectivamente. Para $\mathrm{N}=194 \mathrm{y}$ una proporción muestral de $=.49$ se obtuvo un valor de probabilidad en el análisis de los datos de $\mathrm{p}>.05$, a un nivel de confianza del $95 \%$, por lo cual se aceptó la hipótesis nula.

Para revisar los factores (variables independientes) del perfil psicográfico, que tienen efecto sobre la variable considerada en la hipótesis 4 , después de algunos estudios de correlación, se seleccionaron para un análisis de varianza los factores I3 e I6, "condición laboral" y "estudios de postgrado finalizados", respectivamente. Al considerar todos los ítems de la hipótesis 4, se utilizó un modelo bifactorial y sin interacción, debido a que algunas combinaciones de niveles de los factores no existen, por ejemplo contratado con doctorado. Los resultados se muestran en el Cuadro 3

Cuadro 3. Análisis de Varianza Hipótesis 4 y Factores Condición Laboral y Estudios de Postgrado Finalizados

\begin{tabular}{|c|c|c|c|c|c|}
\hline Fuente & $g l$ & $S C$ & $C M$ & $F$ & $p$ \\
\hline Cond. lab. & 1 & 2.004 & 2.004 & 1.39 & $\begin{array}{c}239 \\
9\end{array}$ \\
\hline $\begin{array}{l}\text { Estudios } \\
\text { Postgrado } \\
\text { Finalizado } \\
\text { s }\end{array}$ & 4 & 27.57 & $\begin{array}{c}6.892 \\
7\end{array}$ & $\begin{array}{c}4.78^{+} \\
+\end{array}$ & $\begin{array}{c}.001 \\
1\end{array}$ \\
\hline Residuos & $\begin{array}{c}18 \\
8\end{array}$ & $\begin{array}{c}270.9 \\
7\end{array}$ & $\begin{array}{c}1.441 \\
3\end{array}$ & & \\
\hline
\end{tabular}

${ }^{++} P<.01$

Dado que $\mathrm{p}>.05$ del factor I3 "condición laboral", no tiene efecto estadístico significativo al nivel de confianza del 95\%; pero $\mathrm{p}<0.05$ para el factor I6 "estudios de postgrado finalizados", por lo tanto este factor (variable independiente) tiene efecto altamente significativo sobre la variable NTIC para el mejoramiento de la calidad en la docencia, al nivel de confianza del $95 \%$.

Las pruebas post hoc de contraste múltiple de rangos, HSD de Tukey para determinar los niveles del factor I6 "estudios de postgrado finalizados" que producen diferencias significativas en los resultados, sobre la variable NTIC como herramienta para mejorar

la calidad en la docencia (ver Cuadro 4).

Conclusión: existen diferencias significativas de todos los primeros cuatro niveles del factor con el nivel 5, y del nivel 1 con el cuatro, y son positivas. Interpretación los últimos niveles de estudios de postgrado no creen mucho en las NTIC como herramienta de mejoramiento de calidad en la docencia, explicable, son razones de tipo generacional.

Cuadro 4. Contraste Múltiple de Rangos para Hipótesis 4 y Factor Estudios de Postgrado Finalizados

\begin{tabular}{lcc}
\hline Contraste & Diferencias & +/- Límites \\
\hline $\begin{array}{l}\text { Diplo. vs. } \\
\text { Docto. (1- } \\
\text { 4) }\end{array}$ & $3.84674^{+}$ & 1.54256 \\
Diplo. vs. & & \\
Postdo. & $3.75583^{+}$ & 1.54256 \\
(1- 5) & & \\
$\begin{array}{l}\text { Especia. } \\
\text { vs. }\end{array}$ & & \\
Postdo. & $2.69639^{+}$ & 1.47689 \\
$(2-5)$ & & \\
Maestría \\
vs. \\
Postdo. & $2.50666^{+}$ & 1.38017 \\
$(3-5)$ & & \\
Doct. vs. & & \\
Postdo. $(4-$ & $1.90909^{+}$ & 1.54256 \\
$5)$ & & \\
\hline${ }^{+} p<.05$ & &
\end{tabular}

De manera similar se procedió con todas las preguntas y se comprobaron la mayoría de las hipótesis de trabajo.

\section{DISEÑO E IMPLEMENTACIÓN DEL SISTEMA}

A partir de los resultados obtenidos en el diagnóstico realizado, del análisis de cualitativo de las respuestas de las entrevistas semiestructuradas; del análisis cuantitativo de la información de las encuestas diagnostico aplicada a los profesores y estudiantes, de la interpretación de los resultados, que dieron respuestas a las preguntas de investigación, a través de los contrastes de hipótesis, de las referencias bibliográficas y de la fundamentación teórica; se procedió al diseño 
del sistema y finalmente se implementó, en un departamento académico de carrera.

\section{Formulación de Indicadores}

Este proceso se explota en diagramas de contexto y otro de procesos de primer orden. Para la obtención de la información que permitió la construcción de los indicadores, se utilizaron diferentes fuentes: profesores y estudiantes con encuestas, Coordinación de Control y Evaluación y Dirección de Recursos Humanos.

Los indicadores resumen para definir el perfil del docente, medir su calidad y productividad, de calidad obtenidos de las variables medidas a través de la encuesta aplicada usando la Web, donde se genera un indicador promedio de los promedios de calidad y productividad, que miden las mismas globalmente y los indicadores promedio por componentes.

Por otro lado, se generó un indicador promedio de calidad de los formatos construidos con la base de datos de la Dirección de Recursos Humanos (DRH) y, para el constructo productividad con la base académica proveniente de la Coordinación de Control y Evaluación.

Ejemplo partiendo de los datos para el profesor número, se construyeron los indicadores, que se observan cuadro 5

Cuadro 5. Indicadores por Componentes de la Calidad y Productividad en la Docencia para el Profesor Número 1

\begin{tabular}{lccccc}
\hline Componente & $I P r$ & $I G C$ & $I G P$ & IpCE & IcDRH \\
\hline Planificación & 2.28 & 2.29 & 2.27 & & \\
Mejoramiento & & 2.24 & 2.22 & & \\
Profesional & 2.23 & & & & \\
Desarrollo y & & 2.20 & 2.15 & & \\
Gestión & 2.17 & & & & \\
Evaluación & 2.22 & 2.20 & 2.23 & & \\
Ético e & & 2.24 & 2.26 & & \\
Interpersonal & 2.25 & & & & \\
Liderazgo & 2.36 & 2.40 & 2.33 & & \\
Investigación & 2.40 & 2.40 & 2.40 & & \\
NTIC & 2.31 & 2.40 & 2.22 & & \\
Total & & 2.24 & 2.21 & 1.89 & 3.00 \\
\hline
\end{tabular}

Nota. IPr $=$ índice promedio; IGC = índice general de calidad; IGP = índice general de productividad.
Todos los indicadores de calidad son provenientes de los de las clases entrada y proceso; y los de productividad provenientes de las clases de insumo y producto, se ponderaron con pesos específicos de acuerdo a la cantidad de elementos y a su tipo y se transformaron en escala de 1 a 3. Para el caso de las variables incluidas en las encuestas se compactaron en tres categorías; los de Control de Estudios se llevaron a la escala de 1 a 100, para luego expresarlos en escala de 1 a 3; y los de la Dirección de Recursos Humanos se categorizaron, en los mismos tres niveles; para generar una escala homogénea. De esta forma se ubicaron dentro de los tres intervalos (subintervalos) generados del intervalo [1, 3], dados por: $[1.0,1.7)$; $[1.7,2.4)$ y $[2.4,3.0]$. Este se transforma en un indicador cualitativo o categorizado; el primero, nivel bajo; el segundo, nivel medio y; el tercero nivel alto. Este proceso se realiza para los indicadores por componente $\mathrm{y}$ totales, de calidad $\mathrm{y}$ productividad.

La ponderación utilizada para la contribución final al cálculo del indicador total fue: $10 \%$ para IcDRH y $30 \%$ para cada uno de los tres restantes IGC, IGP, IpCE; lo que permitió obtener un indicador cuantitativo total para cada profesor ITprof1 a ITprof13. La regla para la toma de decisiones se la siguiente: los profesores ubicados en el nivel bajo deben iniciar un plan de formación o mejoramiento continuo en los cinco componentes de más baja ponderación de índice promedio (IPr) de manera inmediata; los ubicados en el nivel medio deben iniciar un plan de formación o mejoramiento continuo en los tres componentes de más baja ponderación de índice promedio (IPr) en el semestre próximo; y los de nivel alto continuar el plan de formación o mejoramiento continuo que han venido realizando.

\section{Interpretación de Resultados} nero-Diciembre 20 SSN 1794-8231 PP: $102-116$

Así de esta manera con suficiente información, y una base científica, que se genera de todos los estimadores de los indicadores por componente y por tipo, una nueva base de datos cuantitativos y cualitativos, lo cual 
No. 1

Enero-Diciembre 2014 ISSN 1794-8231

PP: $102-116$ facilita la toma de decisiones en cuanto a la formación o el mejoramiento continuo del profesor, en cada uno de los elementos del constructo docencia. Así por ejemplo en el caso del profesor $1 \mathrm{su}$ ITprof1 $=2.20$, lo ubica en el nivel medio, esto sugiere que el profesor debe iniciar en el semestre próximo su plan de formación o mejoramiento continúo en los componentes de docencia con los indicadores IPr más bajos, que en este caso corresponden a: desarrollo y gestión, evaluación, y mejoramiento profesional.

\section{Generación del Perfil del Profesor}

Con base en los resultados de la estimación de los indicadores mostrados en el Cuadro 5, se genera el perfil que se muestran en el gráfico 5 , para el profesor número.

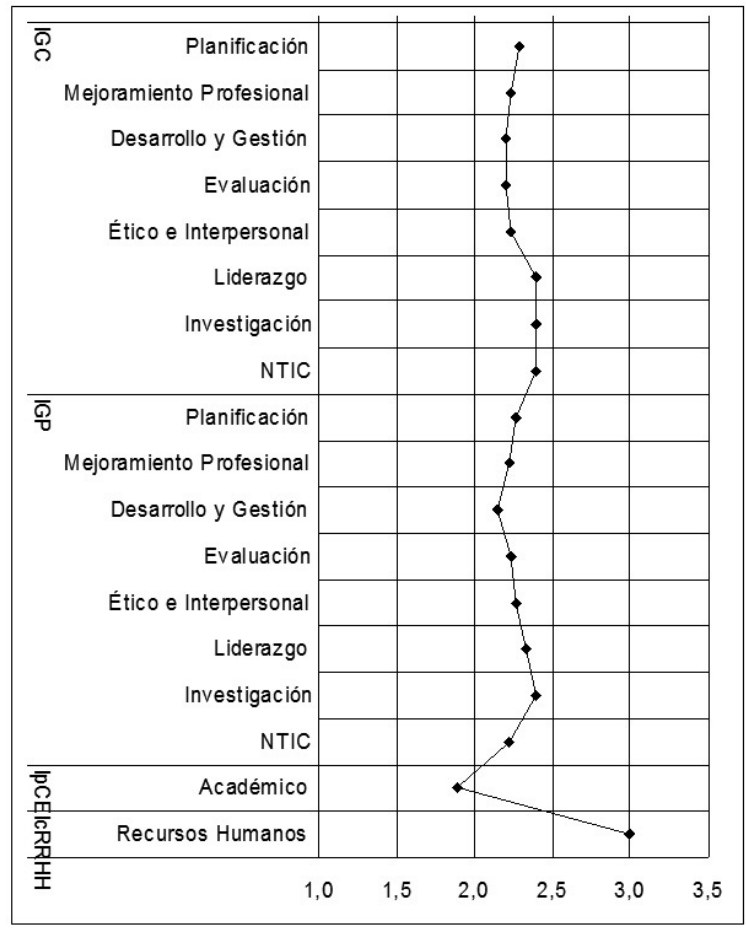

Gráfico 6. Perfil del profesor 1.

\section{CONCLUSIONES Y \\ RECOMENDACIONES}

1. La estructuración de los constructos de docencia, calidad y productividad en la docencia, fue acertada como se confirmó en el análisis factorial. La docencia se conformó con base en ocho componentes; en relación con la calidad en la docencia y productividad, se comprobó la multidimensionalidad de los conceptos; adicionalmente sobre productividad se verificó la ausencia casi total del manejo de este tópico en la educación.

2. Se verificó el desconocimiento de los componentes de la docencia, del manejo de los indicadores de calidad y productividad, y la necesidad de contar con un sistema de medición.

3. De la revisión bibliográfica se detectó la carencia en el país de un sistema de medición de la calidad y la productividad en la docencia.

4. Los análisis de varianza realizados evidenciaron el escaso efecto que tienen los factores más importantes del perfil psicográfico, condición laboral, y estudios de postgrado finalizados sobre la docencia, su calidad, y su productividad.

5. Los estudiantes manifestaron en las dos mediciones realizadas, su interés por contar con un sistema de medición de la calidad y productividad en la docencia.

6. Los procesos de intervención a los cuales son sometidos los profesores contratados en la universidad anfitriona de la investigación, no mejoran los niveles de calidad y productividad en la docencia.

7. El diseño del sistema permitió utilizar una técnica actualizada denominada análisis estructurado, el cual ejecuta el diseño en dos fases: mapas de contexto y mapa general de procesos.

8. La implementación del sistema solo permitió medir indicadores de gestión: entrada, insumo, proceso y producto, son los que tienen que ver más directamente con la misión de la institución.

9. Los resultados generados por el sistema permiten ubicar al profesor en uno de los niveles, bajo, medio, y alto. Sugiriendo las decisiones para cada caso. 
10. La consolidación de un sistema de medición de este tipo, ocurre a mediano plazo como un sistema automatizado inteligente que conduzca en el futuro a la construcción de índices a fin evaluar individual $y$ colectivamente los procesos y resultados, para el mejoramiento continuo de la calidad y la productividad en la docencia.

11. La utilización de las NTIC que permitan la automatización y el uso del ambiente Web para la medición, garantizan mayor participación de usuarios como evaluadores, por asegurar la confidencialidad y el anonimato; es decir, abona hacia mayor objetividad y transparencia al proceso dado que se independiza de las personas.

12. La docencia no es una actividad reducida a la enseñanza en el aula, es todo un sistema que a su vez está constituido por una serie de subsistemas, que interactúan para contribuir al mejoramiento continuo de su calidad y productividad.

13. Este sistema puede contribuir de manera significativa para la medición de la calidad de los procesos y resultados, por dependencias, e institucionalmente, así, de esta forma contribuir a la evaluación y la entrega de cuentas institucionalmente.

\section{RECOMENDACIONES}

A continuación se sugieren una serie de recomendaciones que permitirán actualizar, ampliar y convertir el sistema en un sistema inteligente y extrapolable para otras instituciones de educación superior:

1. Someter a discusión en plenaria y por departamentos el sistema.

2. Someter a discusión los constructos de calidad y productividad en la docencia.

3. Someter a discusión el sistema de indicadores para ir actualizándolo y mejorarlo.

4. Estandarizar el sistema a todas las funciones básicas de la universidad, así como en las actividades financieras y de gestión institucional, para generar indicadores e índices que respondan a los requerimientos del sistema SEA y a los propios de la universidad.

Enero-Diciembre 2014 ISSN 1794-8231 PP: $102-116$

\section{ASPECTOS A DESTACAR EN LA INVETIGACIÓN}

1. Se implementa el teorema de la diversidad complementaria, respecto a la complementariedad de los dos enfoques cuantitativo y cuslitativo.

2. Los ocho constructos o componentes de la calidad y productividad que se generan por el enfoque cuantitativo con la verificación de las hipótesis, a través del análisis descriptivo e inferencial, son los mismos que se generan a través del análisis cualitativo, con las categorías, lo que permite la construcción del sistema de indicadores.

3. El ajuste de la escala de medida a través de la raíz cuadrada para evitare el sesgo y genera una escala de tres valores.

4. La utilización de la estimación por intervalos de confianza para el coeficiente alpha de Crombach, en los construtos para la generación de indicadores.

5. La utilización del coeficiente de confiabilidad parcial por constructo y el coeficiente total.

6. La transformación de un resultado cuantitativo en los indicadores promedio, en un juicio o resultado cualitativo para la toma ce decisiones.

7. Las técnicas de recolección de información de la encuesta a los alumnos y profesores, y la entrevista semiestruturada a expertos y a profesores por grupos en cuanto al tiempo de permanencia en la institución.

\section{REFERENCIAS}

Acevedo, D. (2004). Metodología para la generación e interpretación de indicadores de gestión y resultados. Caracas, Venezuela: Ministerio de Educación Cultura y Deportes.

Alarcón, N. \& Méndez, R. (2000). Calidad y 
No. 1

Enero-Diciembre 2014 ISSN 1794-8231

PP: $102-116$

productividad en la docencia de la educación superior. Recuperado el 2 de septiembre de 2003, de http://www.monografias.com/ trabajos10/ponenc/ponenc.shtml

Albornoz, O. (1997). La cuestión de la productividad, rendimiento y competitividad académica del personal docente $y$ de investigación en América Latina y el Caribe. En L. Yarsabal (Ed.), Educación Superior en el siglo XXI: Visión de América Latina y el Caribe (pp. 391-421). La Habana, Cuba: CRESAL/UNESCO.

Beltrán, J. (1998). Indicadores de gestión. Santafé de Bogotá, Colombia: 3R editores.

Campbell, D. \& Stanley, J. (1995). Diseños experimentales y cuasiexperimentales en la investigación social (M. Kitaigoradzki \& J. C. Orries e I. Bars, Trads.). Buenos Aires, Argentina: Amorrortu. (Trabajo original publicado en 1966).

Hernández, R. (2002). Contribuciones al análisis estadístico. Merida, enezuela: Universidad de Los Andes, Facultad de Ciencias Jurídicas.

Hernández, R., Fernández, C. \& Baptista, P. (2001). Metodología de la investigación (2a. ed.) México: Esfuerzo.

Padrón, J. (2000). Seminario de epistemología y educación. Manuscrito no publicado, Universidad Pedagógica Experimental Libertador. Rubio, Venezuela.

Martínez. M. (1999). Método hermenéuticodialéctico en las ciencias de la conducta. Anhtropos, 10(18), 85-111

Rey, D. (2004). La gestión tradicional y la gestión por procesos. Recuperado el 25 de julio de 2005, de http://www.gestiopolis.com/ recursos $4 /$ docs/ ger/gestitra.htm

Rusque, A. (1999). De la diversidad a la unidad en la investigación cualitativa. Caracas, Venezuela: FACES/UCV.

Salkind, N. (1999). Métodos de investigación (3a. ed.). (R. Escalona, Trad.). México,
México: Prentice Hall Hispanoamericana. (Trabajo original publicado en 1998).

Sánchez, J. (2001). Apuntes de estadística. Manuscrito no publicado. Universidad Nacional Experimental del Táchira. San Cristóbal, Venezuela.

Scheaffer, R., Mendenhall, W. \& Ott, H. (1987). Elementos de muestreo. (G. Sánchez \& J. Gómez, Trads.). México, México: Grupo Editorial Iberoamérica. (Trabajo original publicado en 1986). 